\title{
Arterial spin-labeled MRI study of migraine attacks treated with rizatriptan
}

\author{
Yuji Kato $\cdot$ Nobuo Araki $\cdot$ Hiroshi Matsuda $\cdot$ \\ Yasuo Ito $\cdot$ Chihiro Suzuki
}

Received: 2 February 2010/Accepted: 7 April 2010/Published online: 22 April 2010

(C) Springer-Verlag 2010

\begin{abstract}
Spin-tag perfusion imaging is an MRI method that quantitatively measures cerebral blood flow. Compared with conventional perfusion techniques, advantages of this arterial spin-labeling (ASL) include repeatability and the avoidance of intravenous contrast administration. In the present study, we performed an analysis of 3T highfield MRI examinations utilizing ASL perfusion during migraine attacks. A 32-year-old male patient was studied in three situations: during migraine attack within $1 \mathrm{~h}$ postonset, $30 \mathrm{~min}$ after oral administration of rizatriptan $10 \mathrm{mg}$, and attack-free period. Normalized ASL images acquired during migraine attack showed significant relative hypoperfusion in the bilateral median thalamic areas including hypothalamus and significant relative hyperperfusion in the frontal cortex compared to images acquired during the migraine-free state. When normalized ASL images acquired $30 \mathrm{~min}$ after treatment were compared
\end{abstract}

We got poster award with this work in the 14th International Headache Congress in Philadelphia, USA 2009.

Y. Kato $(\bowtie)$

Department of Neurology and Cerebrovascular Medicine,

Saitama International Medical Center, Saitama Medical

University, 1397-1 Yamane, Hidaka, Saitama 350-1298, Japan

e-mail:yujik@saitama-med.ac.jp

N. Araki · Y. Ito

Department of Neurology, Saitama Medical University,

Moroyama, Saitama, Japan

H. Matsuda

Department of Nuclear Medicine, Saitama International Medical

Center, Saitama Medical University, Hidaka, Saitama, Japan

C. Suzuki

Department of Neurosurgery, Neuroscience Center of Suzuki

Neurosurgical Clinic, Kawagoe, Saitama, Japan with those acquired during the attack, relative improvement of perfusion in the bilateral median thalamic areas including hypothalamus was observed. Hypothalamus and its surrounding areas may participate in the pathogenesis in migraine attack.

Keywords Arterial spin labeling · MRI ·

Migraine without aura $\cdot$ Hypothalamus

\section{Introduction}

Migraine pathophysiology is complex and remains to be not fully elucidated. Functional imaging with positron emission tomography (PET) or functional magnetic resonance imaging (fMRI) has enabled further investigation of migraine pathophysiology in recent years [1-5]. However, the dynamic nature of perfusion abnormalities that occur with migraine limits the application of these methods in the evaluation of such patients.

Spin-tag perfusion imaging is an MRI method that quantitatively measures cerebral blood flow (CBF). Compared with conventional perfusion techniques, advantages of this arterial spin-labeling (ASL) include repeatability and the avoidance of intravenous contrast administration. In the present study, we performed an analysis of 3T highfield MRI examinations utilizing ASL perfusion during migraine attacks.

\section{Methods}

A 32-year-old male patient was studied in three situations: during spontaneous migraine attack within $1 \mathrm{~h}$ post-onset, $30 \mathrm{~min}$ after oral administration of rizatriptan $10 \mathrm{mg}$, and 
Fig. 1 Original ASL images with absolute $\mathrm{CBF}$ values during migraine-free state (a), migraine attack (b), and $30 \mathrm{~min}$ after rizatriptan administration (c). Color bar is absolute CBF values with a range of $0-100 \mathrm{ml} / 100 \mathrm{~g} / \mathrm{min}$. Hypothalamic area shows hypoperfusion during migraine attack (arrows) and recovered by rizatriptan administration. Frontal convexity shows hyperperfusion (arrowheads) during migraine attack compared to migraine-free state

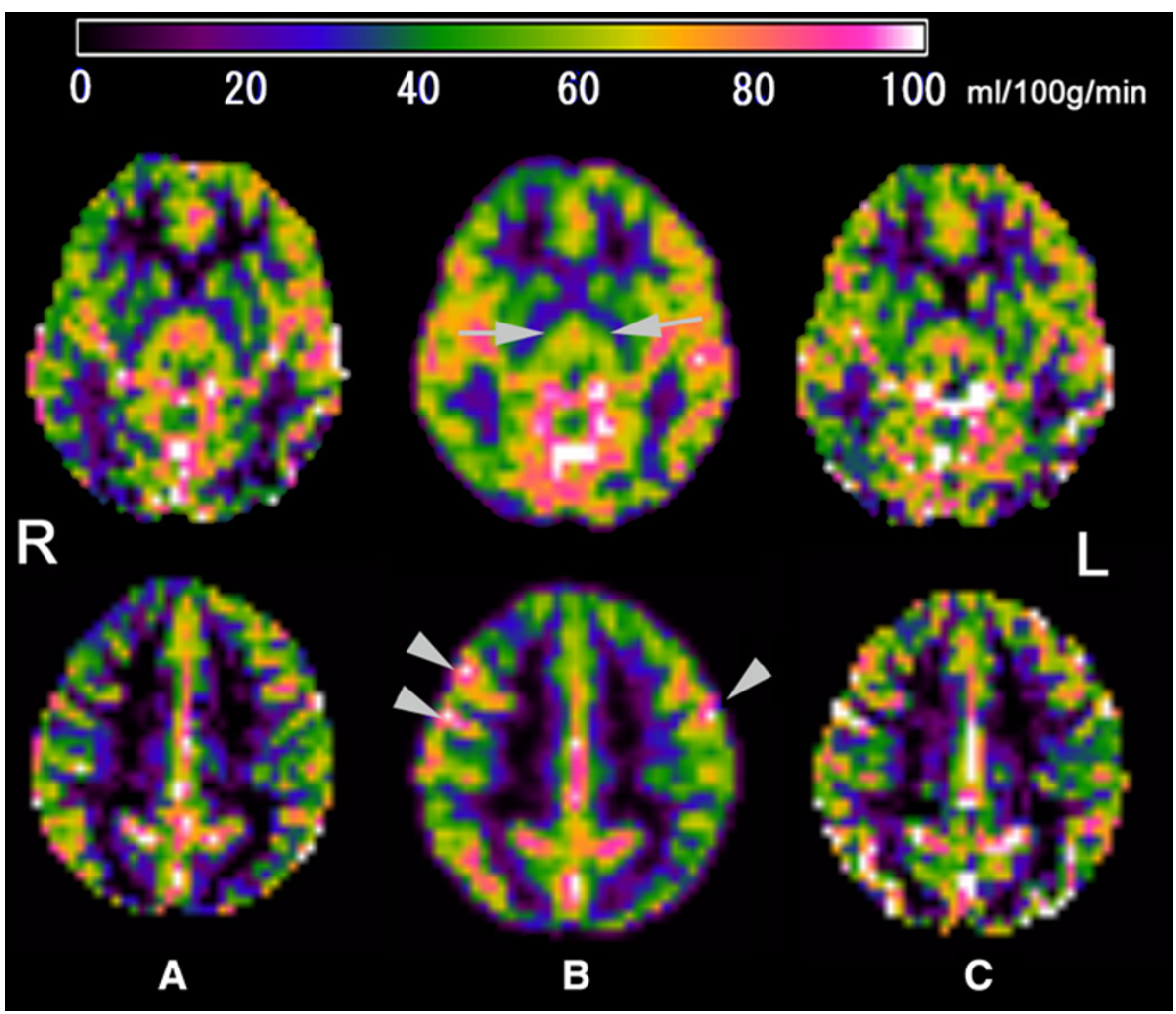

attack-free period. His headache was characterized by moderate to severe, bilateral pulsating headache that was aggravated by physical activity. The attacks were accompanied by loss of appetite, nausea, photophobia, and phonophobia, but neither aura nor hypothalamic symptoms. He fulfilled International Headache Society criteria for episodic migraine without aura (ICHD-II code 1.1). He was not undergoing prophylactic treatment. We performed high-field 3T MR imaging examinations including ASL perfusion at headache attack-free state. We obtained pulsed ASL perfusion images using a technique of QUIPSS II with thin-Slice TI1 periodic saturation as crusher gradients [6]. In the present study, we applied a nuclear medicine technique of subtraction ictal SPECT co-registered to MRI (SISCOM) [7] to analyze relative changes of brain perfusion in different states. All ASL images acquired from the patient during the three states were registered to the MRI scan of the same patient. The co-registered ASL images were normalized according to the global mean voxel CBF. Normalized images in two states were subtracted to obtain images of the difference in CBF between states. Mean and standard deviations of voxel $\mathrm{CBF}$ values were calculated for a subtraction image. The areas with $\mathrm{CBF}$ above two standard deviations in the subtraction image were regarded as significantly increased (warm color scale of $Z$-score) or decreased perfusion (cool color scale of $Z$-score) in a state as compared to another state and superimposed on the tomographic and surface rendering images of the patient's
MRI. This normalization by the global CBF effectively eliminates variations in absolute $\mathrm{CBF}$ measurements and offers more efficient detection of brain perfusion changes in different states than absolute CBF measurements [8].

\section{Results}

Original ASL images with absolute CBF values during migraine attack (Fig. 1a) showed hypoperfusion in bilateral thalamic areas including hypothalamus and hyperperfusion in the frontal convexity compared to images acquired during the migraine state (Fig. 1b). Upon treatment with rizatriptan absolute $\mathrm{CBF}$ in the hypothalamus recovered to the level during the migraine free state (Fig. 1c). These CBF changes are more clearly demonstrated by normalized ASL images with statistical significance. Normalized ASL images acquired during migraine attack showed significant relative hypoperfusion in bilateral thalamic areas including hypothalamus, posterior cingulate, and cerebellum, and relative hyperperfusion in the frontal convexity compared to images acquired during the migraine-free state (Fig. 2a). Upon treatment with rizatriptan, the patient's condition was subjectively assessed to have improved within $30 \mathrm{~min}$. When normalized ASL images acquired 30 min after treatment were compared with those acquired during the attack, relative improvement of perfusion in the hypothalamus and its surrounding areas was observed (Fig. 2b). Cortical 
Fig. 2 a Normalized ASL images during migraine attack show significant relative hypoperfusion in bilateral thalamic areas including hypothalamus, posterior cingulate, and cerebellum, and significant relative hyperperfusion in the frontal convexity as compared to the migraine-free state.

b Normalized ASL images acquired 30 min after rizatriptan administration demonstrated recovered significant relative perfusion in the hypothalamus and its surrounding areas as compared to during migraine attack. a, b Two-tail view. Color bar is Z-score (SD)
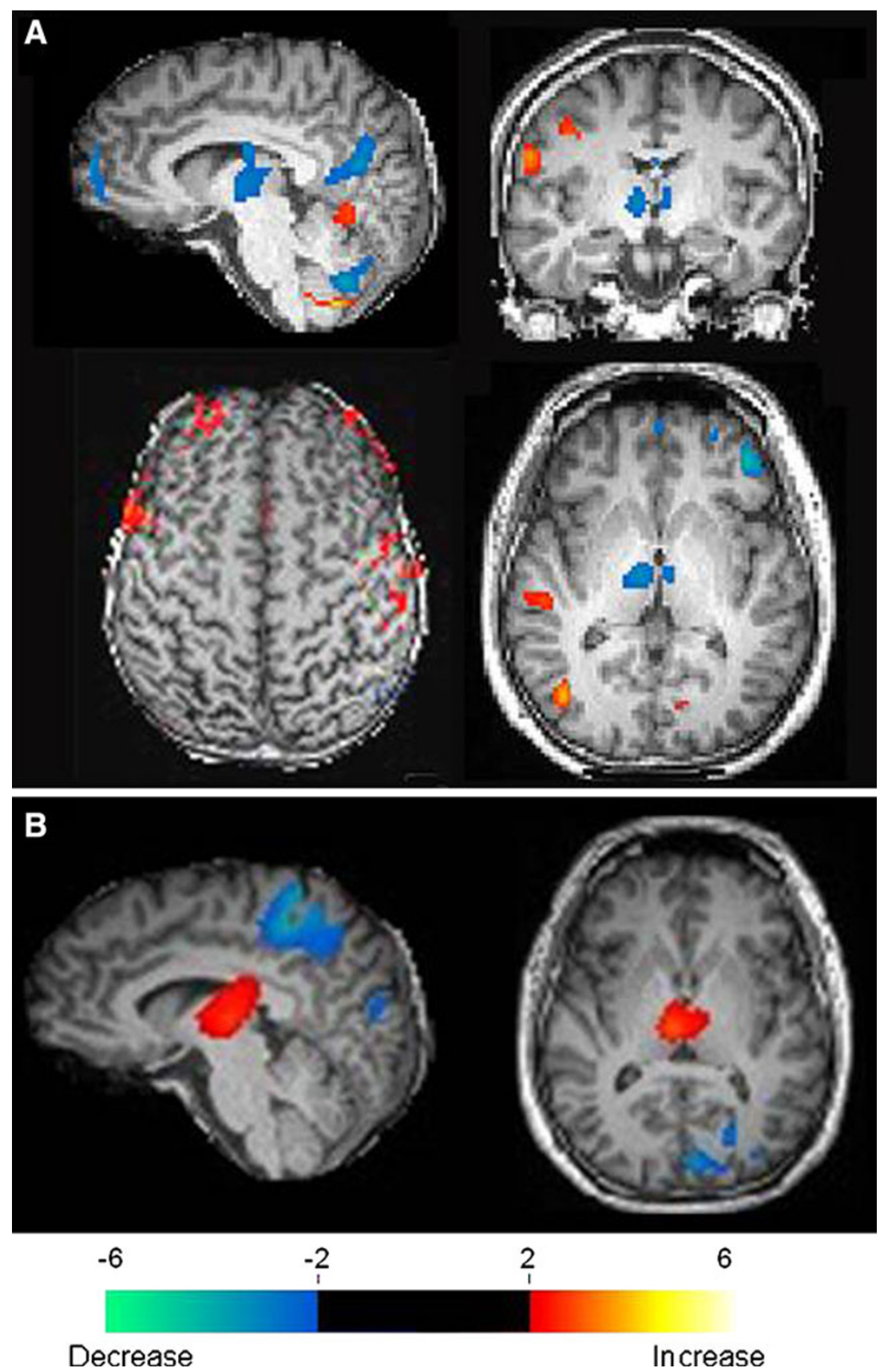

spreading depression (CSD) was not observed in the present study. This patient was studied in two episodes of migraine, the cerebral hemodynamic change was reproducible in bilateral thalamic areas including hypothalamus.

\section{Discussion}

The main difficulty with functional imaging in an episodic disorder is capturing irregular, spontaneous attacks as the imaging techniques require considerable planning. This issue explains the scarcity of functional imaging acquired during spontaneous attacks of migraine. To our knowledge, there is only two cases that have been reported to date of ASL imaging obtained during migraine attack [9, 10], which showed only regional hyperperfusion during the attack. Our report compared findings during migraine attack with the migraine-free state and after administration of rizatriptan.

The findings in the present study were the hypoperfusion of bilateral thalamic areas including hypothalamus, hyperperfusion of the frontal cortex during migraine attacks and rizatriptan appeared to recover the hypoperfusion in the hypothalamus. These data suggested that a functional 
disturbance of the hypothalamus may participate in the pathogenesis in migraine attack, as CBF is closely coupled to neural metabolism [11]. It has long been hypothesized that the prodromal phase of migraine attacks are initiated by a functional disturbance of the hypothalamus, and that either an episodic disturbance of hypothalamic activity or a labile activation threshold could account for the periodicity of the migraine attack [12, 13]. Prior PET studies have shown persisting activation in the brain stem $[1,3]$ or hypothalamus [4] during migraine attack and headache resolution. Then brain stem nuclei may participate in migraine pathogenesis, probably in a dysfunction mode of the anti-nociceptive network and cerebrovascular control. On the other hand, only few studies have found hypoperfusion or deactivation [3, 5, 14]. Shazia et al. [3] reported deactivation in the thalamus and the insula. Denuelle et al. [5] reported posterior cortical hypoperfusion similar to CSD during migraine without aura. These discrepancies of perfusion may be caused by the timing of the scans or the imaging techniques. Our patient was studied within $1 \mathrm{~h}$ post-onset, which was much earlier phase than previous studies. Hypoperfusion in our case could possibly be prodromal phase of hyperactivation or transitional period to CSD. We need to consider the significance of not only the hyperperfusion, but also the hypoperfusion in migraine attack.

Although previous study has shown that rizatriptan decreases global and regional values of CBF in healthy volunteers [15], the findings of the present study indicated that rizatriptan seemed to recover hypothalamic CBF reduction. Although we do not know hemodynamic change in hypothalamus is specific to migraine or non-specific pain process [16], this is the first case in which hemodynamic change in hypothalamus was detected using ASL during migraine attack treated with triptan. If the hypothalamus does constitute the generator and integrator of migraine attack, this must be confirmed by sequential neuroimaging of spontaneous attacks from the prodromal phase onward in more patients.

Conflict of interest None.

\section{References}

1. Weiller C, May A, Limmroth V, Jüptner M, Kaube H, Schayck RV, Coenen HH, Diener HC (1995) Brainstem activation in spontaneous human migraine attacks. Nat Med 1:658-660
2. Cao Y, Aurora SK, Nagesh V, Patel SC, Welch KMA (2002) Functional MRI-BOLD of brainstem structures during visually triggered migraine. Neurology 59:72-78

3. Shazia K, Afridi SK, Giffin NJ, Kaube H, Friston KJ, Ward NS, Frackowiak RS, Goadsby PJ (2005) A positron emission tomography study in spontaneous migraine. Arch Neurol 62:1270-1275

4. Denuelle M, Fabre N, Payoux P, Chollet F, Geraud G (2007) Hypothalamic activation in spontaneous migraine attacks. Headache 47:1418-1426

5. Denuelle M, Fabre N, Payoux P, Chollet F, Geraud G (2008) Posterior cerebral hypoperfusion in migraine without aura. Cephalalgia 28:856-862

6. Luh WM, Wong EC, Bandettini PA, Hyde JS (1999) QUIPSS II with thin-slice TI1 periodic saturation: a method for improving accuracy of quantitative perfusion imaging using pulsed arterial spin labeling. Magn Reson Med 41:1246-1254

7. Matsuda H, Matsuda K, Nakamura F, Kameyama S, Masuda H, Otsuki T, Nakama H, Shamoto H, Nakazato N, Mizobuchi M, Nakagawara J, Morioka T, Kuwabara Y, Aiba H, Yano M, Kim YJ, Nakase H, Kuji I, Hirata Y, Mizumura S, Imabayashi E, Sato N (2009) Contribution of subtraction ictal SPECT coregistered to MRI to epilepsy surgery: a multicenter study. Ann Nucl Med 23:283-291

8. Yoshiura T, Hiwatashi A, Noguchi T, Yamashita K, Ohyagi Y, Monji A, Nagao E, Kamano H, Togao O, Honda H (2009) Arterial spin labelling at 3-T MR imaging for detection of individuals with Alzheimer's disease. Eur Radiol 19:2819-2825

9. Pollock JM, Deibler AR, Burdette JH, Kraft RA, Tan H, Evans AB, Maldjian JA (2008) Migraine associated cerebral hyperperfusion with arterial spin-labeled MR imaging. AJNR Am J Neuroradiol 29:1494-1497

10. Pollock JM, Tan H, Kraft RA, Whitlow CT, Burdette JH, Maldjian JA (2009) Arterial spin-labeled MR perfusion imaging: clinical applications. Magn Reson Imaging Clin N Am 17:315338

11. Jueptner M, Weiller C (1995) Review: does measurement of regional cerebral blood flow reflect synaptic activity? Implications for PET and fMRI. Neuroimage 2:148-156

12. Lance JW (1989) Headache: classification, mechanism and principles of therapy, with particular reference to migraine. Recenti Prog Med 80:673-680

13. Silberstein SD (1992) Advances in understanding the pathophysiology of headache. Neurology 42(suppl 2):6-10

14. Woods RP, Iacoboni M, Mazziotta JC (1994) Brief report: bilateral spreading cerebral hypoperfusion during spontaneous migraine headache. N Engl J Med 331:1689-1692

15. Okazawa H, Tsuchida T, Pagani M, Mori T, Kobayashi M, Tanaka F, Yonekura Y (2006) Effects of 5-HT1B/1D receptor agonist rizatriptan on cerebral blood flow and blood volume in normal circulation. J Cereb Blood Flow Metab 26:92-98

16. Peyron R, Laurent B, Garcia-Larrea L (2000) Functional imaging of brain responses to pain. A review and meta-analysis. Neurophysiol Clin 30:263-288 